\title{
Harnessing non-covalent interactions towards the isolation of novel actinide structural units
}

\author{
Karah E. Knope, \\ Georgetown University, Washington DC, USA \\ kek44@georgetown.edu
}

Counterions are ubiquitous in solution but the role they play in species formation, stability, and reactivity is not well understood. Inspired by recent work that has shown that consideration of counterions may be important for understanding phase formation and the overall chemical behavior of a metal ion, our group has sought to examine the impact of nonbonding interactions on actinide (An) complex formation and precipitation. Our efforts have focused on the solution and solid-state structural chemistry of An-Cl complexes formed from acidic aqueous chloride solutions in the presence of protonated N-heterocycles. Within this context, a series of seven unique $\mathrm{Th}^{\mathrm{IV}}$ compounds that were precipitated from aqueous solution will be presented. The compounds consist of $\mathrm{Th}^{\mathrm{IV}} \mathrm{metal}^{\mathrm{c}}$ centers that adopt 8- or 9-coordinate complexes with the general formulas $\left[\mathrm{Th}\left(\mathrm{H}_{2} \mathrm{O}\right)_{x} \mathrm{Cl}_{8-x}\right]^{\mathrm{x}-4}(x=2,4)$ and $\left[\mathrm{Th}\left(\mathrm{H}_{2} \mathrm{O}\right)_{x} \mathrm{Cl}_{9-x}\right]^{\mathrm{x}-5}(x=5-7)$. While all of the complexes are heteroleptic, bound to $\mathrm{Cl}^{-}$and $\mathrm{H}_{2} \mathrm{O}$ ligand, the structural units vary in composition, charge, and coordination geometry. The complexes range from chloride rich to chloride deficient, with the number of bound chlorides and hence charge on the structural unit showing some dependence on the counterion present in the outer coordination sphere. Our experimental and computational efforts to understand phase formation, the effects of noncovalent interactions, and the energetics that drive the formation of this series of structurally related $\mathrm{Th}^{\mathrm{IV}}$-aquo-chloro compounds will be discussed.

Keywords: structural chemistry, actinides 